\title{
Expression Of Androgen Receptor, Estrogen Receptor And Progesterone Receptor In Endometrial Carcinoma (Immunohistochemical Study)
}

\author{
Suruchi Mangal ${ }^{1}$, Manisha Sharma ${ }^{1}$, Mridu Manjari ${ }^{1}$, Rahul Mannan ${ }^{1}$ and Sunit Tandon ${ }^{2 *}$ \\ ${ }^{1}$ Dept of pathology, Sri Guru Ram Das Institute of Medical Sciences and Research, Vallah, Amritsar, India \\ ${ }^{2}$ Dept of Surgery, GMC Amritsar, India
}

\begin{abstract}
Background: Endometrial carcinoma is the most common pelvic genital malignancy and 4th most frequently diagnosed cancer in females with it's incidence being 4.3/ 1 lac females.

Methods: Thirty histologically proven cases of endometrial carcinoma were taken up for the study in pathology department. Immunohistochemistry for expression of androgen receptor (AR), estrogen receptor (ER) and progesterone receptor (PR)was done using Biocare system kit.

Results: $80 \%$ of the cases were in 5 th and 6 th decade of life. Bleeding per vagina and post menopausal bleeding were the main complaints. Out of the total 22 abdominal hysterectomies the size of tumor varied from $1 \mathrm{~cm}$ to $>4 \mathrm{~cm}$. All were adenocarcinoma with 20 being moderately differentiated and only 2 being well differentiated.

AR was positive in 8 cases with ER and PRbeing positive in 16 and 21 cases respectively with score being also the same. Receptor positivity decreased with increasing grade of the tumor.

Conclusion: ER and PR status are important prognostic biomarkers which also predict response to antihormonal therapy in endometrial carcinoma. AR expression though associated with low grade tumors, but still is a driver for tumor growth and therefore a potential therapeautic target. Anti androgen therapy - enzalutamide may inhibit proliferation of AR positive primary endometrial cancer cells.
\end{abstract}

Keywords: Endometrial Adenocarcinoma, Androgen Receptor, Estrogen Receptor, Progesterone Receptor

\section{Introduction}

Endometrial carcinoma is the most common female pelvic genital malignancy and the $4^{\text {th }}$ most frequently diagnosed cancer in women especially in the developed world, with an increasing incidence related to obesity. There is a strong association between development of endometrial cancer and the influence of steroid hormones (especially estrogen). ${ }^{[1]}$ In developed countries like USA it's incidence is 25.7 per $1,00,000$ with mortality of $2.1 \%$. Whereas in India it's incidence is as low as 4.3 per $1,00,000$ with mortality of $1.5 \%$. It mainly occurs in females aged 55-64 years with an estimated 61,380 new cases and 10,920 deaths in 2017. ${ }^{[2]}$

There are two types of endometrial cancer on the basis of pathogenesis:-Type I endometrial carcinomas which represents $75-90 \%$ of endometrial cancer. They are associated with endometrioid histology, low-grade, minimally invasive into the myometrium, estrogen dependent and have a good outcome with treatment. Type II endometrial carcinomas are associated with non endometrioid histology, high stage and grade and poor prognosis, with a greater risk of relapse and/or metastasis ${ }^{[3]}$

Development of endometrial carcinoma has been linked to multiple factors: hormonal factors, constitutional factors and genetic factors. The risk of endometrial cancer is positively correlated with older age, early menarche \& late menopause, obesity, family history of endometrial cancer (especially among close relatives), exposure to radiation, and infertility particularly in the presence of Polycystic Ovarian Syndrome. The risk of endometrial cancer also increases with longterm use of unopposed estrogens as hormone replacement therapy. ${ }^{[4]}$

The various modalities used for its diagnosis are : Endometrial biopsy, Dilation and curettage, Hysteroscopy, Papanicolaou (Pap) Smear, Imaging studies, Molecular markers, cytogenetics, and Immunohistochemistry markers.

In recent years Immunohistochemistry (IHC) in endometrial carcinoma has emanated as an important tool for diagnosis of the type and predicting prognosis.

\section{Material and Methods}

The study was conducted on 30 histopathologicaly proven cases of endometrial carcinoma diagnosed in Department of Pathology, Sri Guru Ram Das Institute of Medical Sciences And Research, Amritsar. Histopathological examination of the tissues obtained was done after processing them 
to prepare paraffin blocks. Blocks were cut and stained with Haematoxylin and Eosin stain and studied under light microscope for classification and histopathological grading.

Immunohistochemistry of the tumors was done for AR, PR and ER using Primary antibody - Mouse Monoclonal Antibody (Biomedical Care). Positive and negative controls were run with every batch of the IHC. For AR, ER and PR nuclear staining was studied and assessed semiquantitatively using Liverpool endometrial steroid quick score (LESQS). ${ }^{[5]} \mathrm{A}$ final immunoscore out of 12 was calculated by multiplying scores of $\%$ and intensity and categorised as positive result $(\geq 2)$ and negative result $(\leq 1)$

\begin{tabular}{|l|l|l|l|}
\hline $\begin{array}{l}\text { Percentage } \\
\text { positivity }\end{array}$ & Score & $\begin{array}{l}\text { Staining } \\
\text { intensity }\end{array}$ & Score \\
\hline$\leq 10 \%$ & 1 & Nil & 0 \\
\hline$>10$ to $\leq 20 \%$ & 2 & Mild & 1 \\
\hline$>20$ to $\leq 40 \%$ & 3 & Moderate & 2 \\
\hline$>40$ & 4 & Severe/Strong & 3 \\
\hline
\end{tabular}

\section{Results}

The majority of the patients were in the age group of 5160 yrs $(53 \%)$. The youngest patient was 30 years old and the oldest was 80 years.(Table 1) Most of the cases (12) presented with post menopausal bleed as chief complaint followed by pain abdomen, bleeding per vaginum, menorrhagia and discharge.(table 2)

Tumor size in 22 hysterectomy cases varied from less than $1 \mathrm{~cm}$ to more than $4 \mathrm{~cm}$.All the endometrial tumors came out to be adenocarcinoma on histopathology. Endometrial adenocarcinoma cases were not further subclassified into endometrioid and non endometrioid.

All the cases were further subdivided based on differentiation (fig 1,2,3). Maximum cases came out to be moderately differentiated (67\%) . (table 3) 08 (27\%) cases showed Androgen Receptor positivity, 16 (53\%)cases showed Estrogen Receptor positivity and 21 (70\%) cases showed Progesterone Receptor positivity (cases showing total score of $\geq 2$ were considered as positive) (fig 4,5,6). Triple positive cases were 06 and triple negative were 08 . Two cases were such which showed ER negativity but were positive for AR and PR.

AR, ER and PR positivity was maximally seen in moderately differentiated endometrial adenocarcinoma cases- 35\%, $60 \%$ and $80 \%$ respectively. Marker positivity decreased with dedifferentiation i.e. it reduced to $13 \%, 38 \%$ and $50 \%$ for AR, ER, PR respectively in poorly differentiated cases. More differentiated the tumor is, better the prognosis ( $p$ value $=0.945)$. (table 4$)$

As the invasion increased marker positivity and intensity reduced with AR and PR (such decrease was not observed with ER may be because of limited number of cases included in the study). Absent myometrial invasion showed 100\% $\mathrm{AR}$ and PR positivity. Whereas the positivity reduced as depth of invasion increased-AR and PR positivity reduced to $11 \%$ and $66 \%$ respectively for cases showing two-third invasion. Hence, as the tumor grows more, prognosis becomes poor $(p$ value $=0.667)$. $($ table 5$)$

Table 1

\begin{tabular}{|c|c|c|}
\hline Age Group & No. of cases & Percentage \\
\hline$<30$ & 01 & 0 \\
\hline $31-40$ & 00 & 7 \\
\hline $41-50$ & 02 & 53 \\
\hline $51-60$ & 16 & 27 \\
\hline $61-70$ & 08 & 10 \\
\hline$>70$ & 03 & \\
\hline
\end{tabular}

Table 2

\begin{tabular}{|c|c|}
\hline CHIEF COMPLAINT & NO. OF CASES \\
\hline Bleeding per vagina & 06 \\
\hline Pain abdomen & 07 \\
\hline Menorrhagia & 04 \\
\hline Post menopausal bleed & 12 \\
\hline Discharge per vagina & 01 \\
\hline
\end{tabular}


Table 3

\begin{tabular}{|c|c|c|}
\hline DIFFERENTIATION & NO. OF CASES & PERCENTAGE \\
\hline Well differentiated & 02 & 7 \\
\hline Moderately differentiated & 20 & 67 \\
\hline Poorly differentiated & 08 & 26 \\
\hline
\end{tabular}

Table 4

\begin{tabular}{|c|c|c|c|c|c|c|c|}
\hline GRADE & No. of cases & \multicolumn{2}{|c|}{ AR } & \multicolumn{2}{c|}{ ER } & \multicolumn{2}{c|}{ PR } \\
\hline WD & 02 & 00 & 00 & 01 & $50 \%$ & 01 & 16 \\
\hline MD & 20 & 07 & $35 \%$ & 12 & $60 \%$ & $80 \%$ \\
\hline PD & 08 & 01 & $13 \%$ & 03 & $38 \%$ & 04 & $50 \%$ \\
\hline
\end{tabular}

Table 5

\begin{tabular}{|c|c|c|c|c|}
\hline MYOMETRIAL INVASION & NUMBER OF CASES & \multicolumn{3}{|c|}{ AR ER PR } \\
\hline ONE-THIRD (33\%) & 10 & $2(20 \%)$ & $4(40 \%)$ & $7(70 \%)$ \\
\hline HALF (50\%) & 01 & $1(100 \%)$ & $1(100 \%)$ & $1(100 \%)$ \\
\hline TWO-THIRD $(66 \%)$ & 09 & $1(11 \%)$ & $6(66 \%)$ & $6(66 \%)$ \\
\hline THREE-FOURTH (75\%) & 01 & 0 & 0 & 0 \\
\hline NO INVASION & 01 & $1(100 \%)$ & 0 & $1(100 \%)$ \\
\hline
\end{tabular}

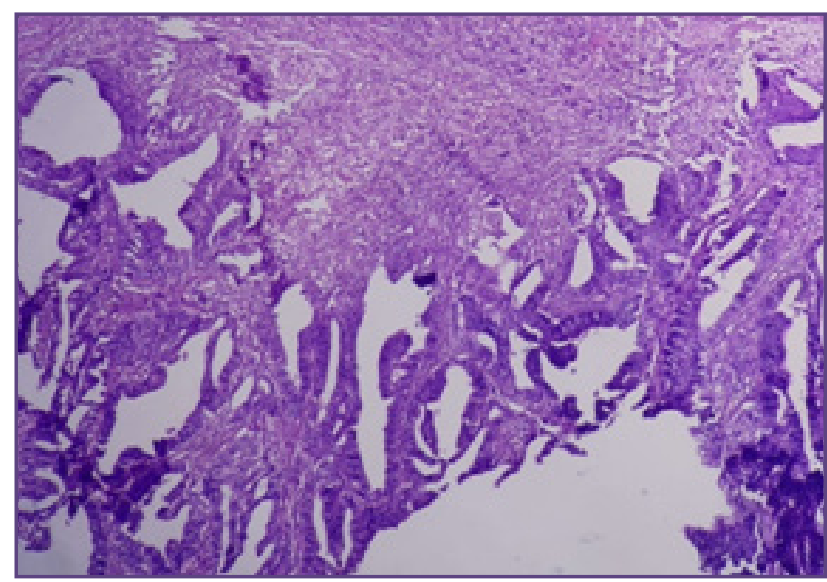

Fig. 1: Well differentiated adenocarcinoma (H and $\mathrm{E}$, 100X)

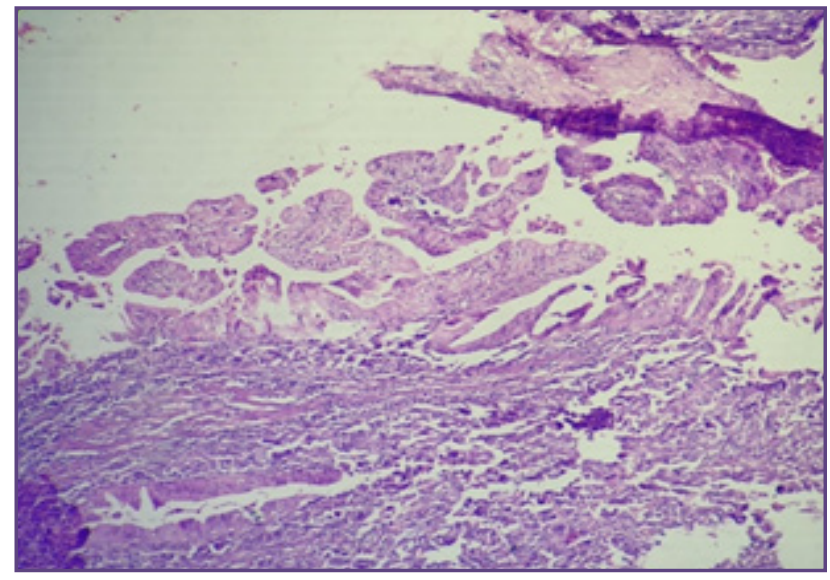

Fig 3: Poorly differentiated adenocarcinoma (H and E, 100X)

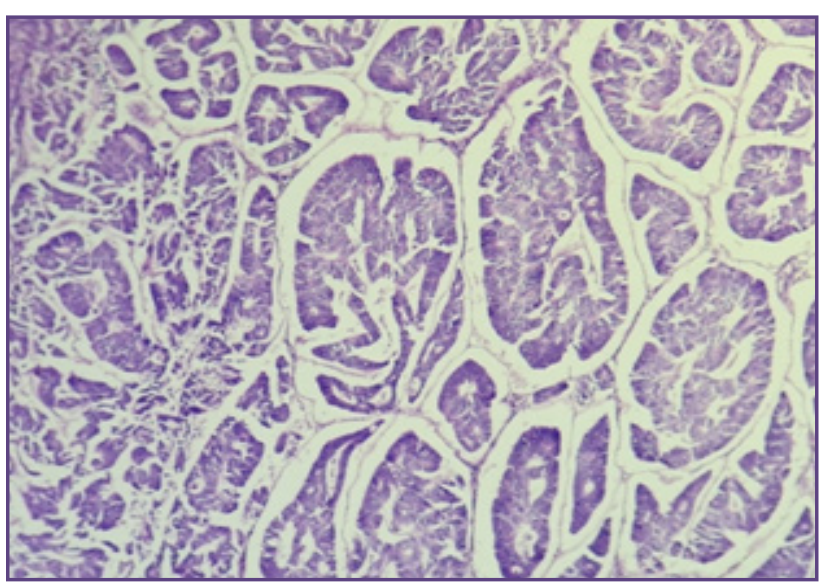

Fig. 2: Moderately differentiated adenocarcinoma ( $\mathrm{H}$ and E, 100X)

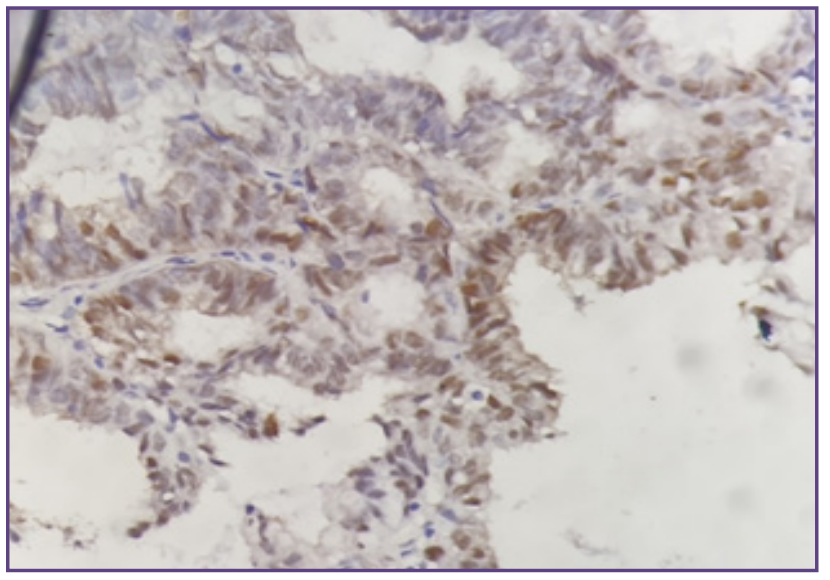

Fig 4: Androgen Receptor Staining (nuclear, moderate intensity, 400X) 


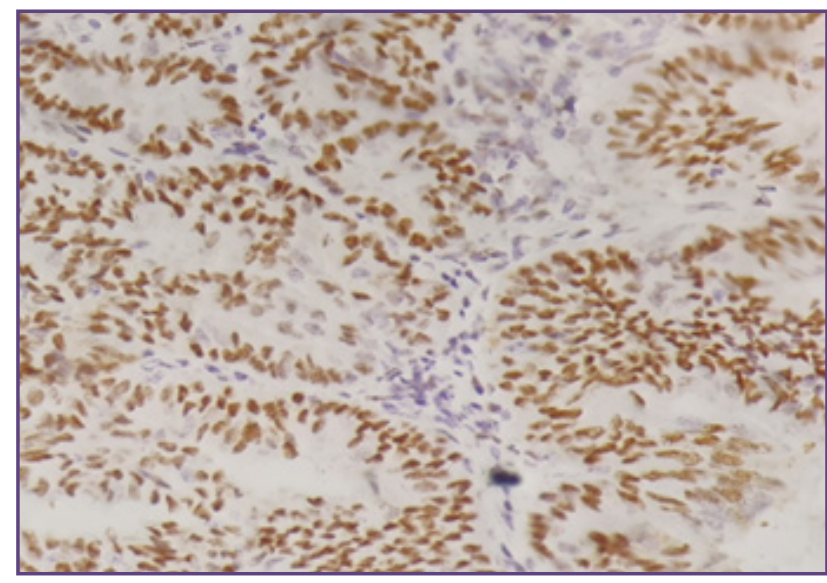

Fig. 5: Estrogen Receptor Staining (nuclear, strong intensity, 400X).

\section{Discussion}

Endometrial carcinoma is the most common female pelvic genital malignancy and the $4^{\text {th }}$ most frequently diagnosed cancer in women especially in the developed world. Its incidence is on the rise mainly because of obesity. ${ }^{[1]} \mathrm{A}$ wide variety of morphology based and molecular based endometrial cancer prognostic factors and tumor markers had been studied to identify the oncogenes involved in initiation and and progression of tumor and development of new anticancer drugs. Role of ER and PR had been extensively studied. Recently AR is being widely explored to know its role in endometrial carcinoma and develop new treatment modalities.

In our study the majority of the patients were in the age group of 51-60 yrs and presented mostly with post menopausal bleed. Maximum cases came out to be moderately differentiated $(67 \%)$ followed by poorly differentiated (26\%) and well differentiated (7\%). In a study conducted by Modi et al most cases of endometrial carcinoma were well differentiated adenocarcinoma (41\%), followed by moderately differentiated (27\%) and then poorly differentiated (10\%). ${ }^{[6]}$ In our study most of the cases were moderately and poorly differentiated may be that patients in our case mostly report late because of illiteracy especially in rural population.

In our study, none of the well differentiated cases showed AR positivity but the percentage positivity for AR in moderately differentiated cases was $35 \%$ which reduced to $13 \%$ in poorly differentiated cases. Similar results had been observed by Tangen et al in their study ${ }^{[3]}$ Kato J in his study also showed decrease in AR positivity with loss of differentiation (highly differentiated tumors $=21$ cases, moderately $=7$ and poorly differentiated $=$ one out of 8$).{ }^{[7]}$

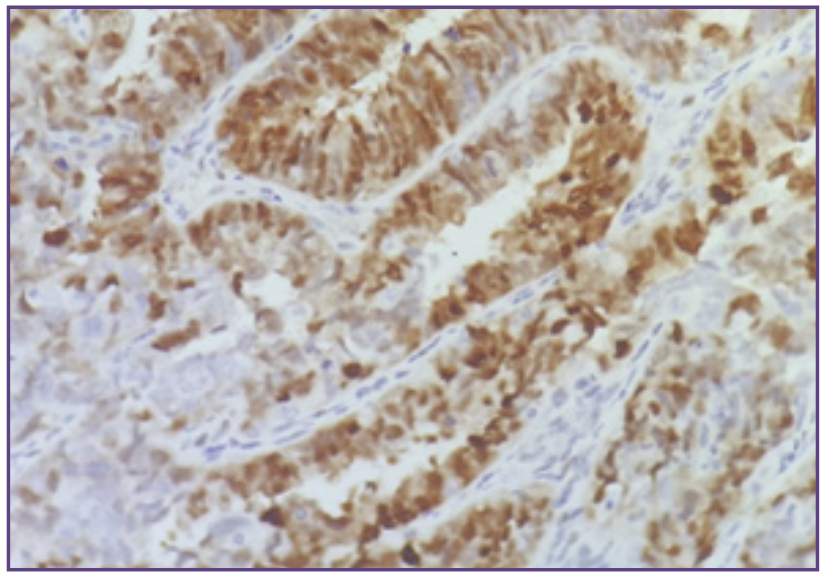

Fig. 6: Progesterone Receptor Staining (nuclear, strong intensity, 400X).

ER, PR expression in our study was $50 \%$ in well differentiated cases for both, followed by $60 \%$ and $80 \%$ for ER and PR respectively in moderately differentiated cases which reduced to $38 \%$ and $50 \%$ for ER and PR respectively in poorly differentiated cases. These results were consistent with previous reports. ${ }^{[8,9,10]}$

Our study showed that case with no myometrial invasion showed $100 \%$ AR and PR positivity. Whereas the positivity reduced as depth of invasion increased-AR and PR positivity reduced to $11 \%$ and $66 \%$ respectively for cases showing two-third invasion. It was not so with ER may be because of limited number of cases included in our study. This was in agreement with the study done by Mahdi et al..$^{[1]} \mathrm{Kamal}$ et al has also evaluated the positive correlation of decrease in $\mathrm{AR} / \mathrm{PR}$ positivity and increase in ER positivity with myometrial invasion. AR/PR expression is associated with longer disease free survival as opposed to ER whose higher levels are associated with shorter disease free survival. ${ }^{[5]}$ In contrast, study by Guan et al showed loss of both ER and PR was associated with deeper invasion, severer FIGO stage and higher rate of pelvic node metastasis..$^{[12]}$

AR, ER and PR status are important prognostic biomarkers which also predict response to antihormonal therapy in endometrial carcinoma. Few cases in our study were positive for AR and PR but negative for ER. Such cases can be benefited by anti androgen therapy alongwith anti hormonal therapy as supported by study conducted by Tangen et al. ${ }^{[3]}$

\section{Conclusion}

Thus it is concluded that as observed from the results ER positivity increased with increasing depth of invasion which was taken as poor prognostic factor.

All the cases which presented with receptor positivity with hormone receptor positivity i.e. ER and PR, they respond 
to hormonal therapy. Whereas, if the cases express AR also they respond better with anti androgen therapy combined with hormonal therapy. Thus showing that all the cases of endometrial carcinoma should be subjected to expression of AR, ER and PR because as reported AR, PR expression is taken as a good prognostic factor as it is associated with longer disease free survival period. Whereas, ER expression is taken as a poor prognostic factor as it is associated with shorter disease free survival period.

\section{Financial support and sponsorship Nil}

\section{Conflicts of Interest}

There are no conflicts of Interest

\section{Bibliography}

1. Shabani N, Mylonas I, Jeschke U, Thaqi A, Kuhn C, Puchner T, et al. Expression of estrogen receptors $\alpha$ and $\beta$, and progesterone receptors $\mathrm{A}$ and $\mathrm{B}$ in human mucinous carcinoma of endometrium. Anticancer Res. 2007 Mar;27:2027-34.

2. National Cancer Institute surveillance, epidemiology and end results program. Cancer stat facts: Endometrial Cancer [Internet]. USA, New York: SEER;2017[updated 2017 Jun 28; cited on 2017 Nov 21]. Available from . https://seer. cancer.gov/ statfacts/html/corp.html.

3. Tangen IL, Onyango TB, Kopperud R, Berg A, Halle MK, Oyan AM, et al. Androgen receptor as potential therapeautic target in metastatic endometrial cancer. Oncotarget [Internet]. 2016 Aug [cited on 2017 Nov 22];7(31):4928998. Available from:https://www.ncbi.nlm.nih.gov/pmc/ articles/PMC5226508/ DOI:10.18632/oncotarget.10334.

4. Tariq AA. Risk factors for Endometrial cancer. Ces. Gynek. 2013;78(5):448-59.

5. Kamal AM, Bulmer JN, De Cruze SB, Stringfellow HF, Martin-Hirsch P, Hapangama DK. Androgen receptors are acquired by healthy postmenopausal endometrial epithelium and their subsequent loss in endometrial cancer is associated with poor survival. Br. J. Cancer [Internet]. 2015 Dec [cited on 2017 Nov28];114:688-96. Available from: https://www. bjcancer.com DOI:10.1038/bjc.2016.16.

6. Modi M, Nilkanthe R, Trivedi M. Detailed Histopathological Study of Endometrial Carcinoma, and Importance of Immunohistochemistry. Am J Clin Path. 2016 Sept;146(1):74-7.

7. Kato J, Seto T. Correlation of androgen receptors with histological differentiation in human endometrial carcinomas. Acta Obstet Gynecol Scand. 1985;64(3):20912.

8. Bender D, Buekers T, Leslie KK. Hormones and Receptors in Endometrial Cancer. Proc Obstet Gynaecol. 2011 Jul;2(1):125.

9. Waqar S, Khan SA, Sarfraz T, Waqar S. Expression of Estrogen Receptors (ER), Progesterone Receptors (PR) and HER-2/neu receptors in Endometrial Carcinoma and their associations with histological types, grades and stages of the tumor. Pak J Med Sci. 2018 Mar;34(2):266-71.

10. McCarty KS, Jr., Barton TK, Fetter BF, Creasman WT, McCarty KS, Sr. Correlation of estrogen and progesterone receptors with histologic differentiation in endometrial adenocarcinoma. Am J Pathol. 1979;96(1):171-83.

11. Mahdi Z, Abdulfatah E, Pardeshi V, Hassan O, Schultz $\mathrm{D}$, Morris R, et al. The Impact of Androgen Receptor Expression on Endometrial Carcinoma Recurrence and Survival. Int J Gynecol Pathol. 2017 Sep;36(5):405-11.

12. Guan J, Xie L, Luo X, Yang B, Zhang H, Zhu Q, et al. The prognostic significance of estrogen and progesterone receptors in grade I and II endometrioid endometrial adenocarcinoma: hormone receptors in risk stratification. J Gynecol Oncol [Internet]. 2019 Jan [cited on 2019 Aug 20]; 30(1): e13. Available from: https://www.ncbi. nlm.nih.gov/pmc/articles/PMC6304404/ DOI: 10.3802/ jgo.2019.30.e13.

*Corresponding author:

Dr. Sunit Tandon, Opposite Government senior secondary school, Tanda, District Hoshiarpur144204 INDIA

Phone: +91 9417741031

Email: suruchi2506@gmail.com

Financial or other Competing Interests: None. 